Supporting Information for:

\title{
A novel rhenium chelate system derived from dimercaptosuccinic acid for the selective labeling of biomolecules
}

Tobias K. Heinrich ${ }^{\dagger}$, Werner Kraus ${ }^{\S}$, Hans-Jürgen Pietzsch ${ }^{*}$, Christoph Smuda ${ }^{\dagger}$, and Hartmut Spies ${ }^{\dagger}$

Forschungszentrum Rossendorf, Institut fuer Bioanorganische und Radiopharmazeutische Chemie, PF 510 119, D-01314 Dresden, Germany,

Bundesanstalt fuer Materialforschung und -pruefung, Lab I.33, Richard-WillstaetterStrasse 11, D-12489 Berlin, Germany

'Institut fuer Bioanorganische und Radiopharmazeutische Chemie.

${ }^{\S}$ Bundesanstalt fuer Materialforschung und -pruefung. 


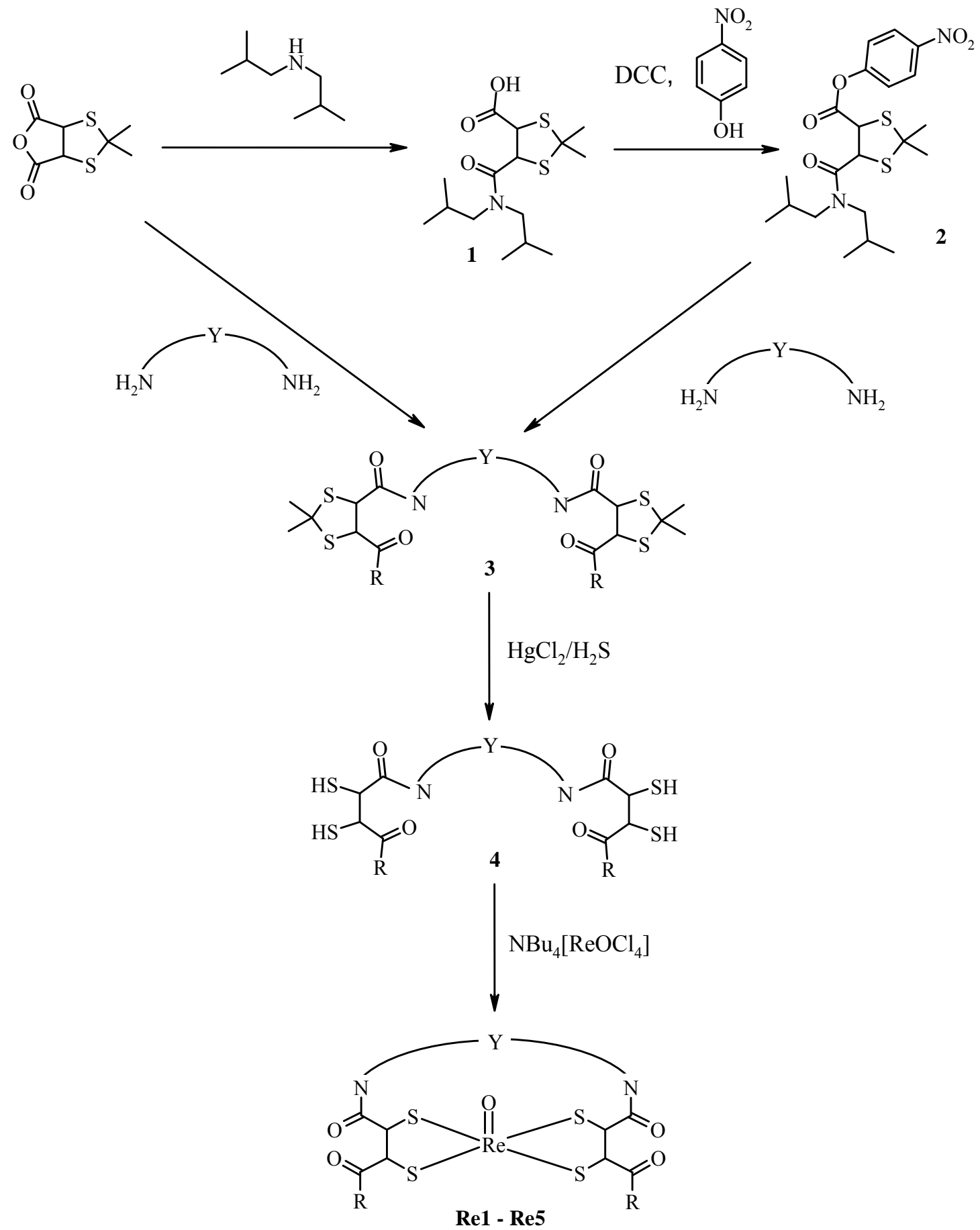

Scheme1. Reaction routes to (amino)alkylene bridged $\mathrm{S}_{4}$ DMSA ligands (4) and rhenium complexes ( $\mathbf{R e} 1-\mathbf{R e} 5)$ 
General procedure for the reaction of 2,2-dimethyl-dihydro-[1,3]dithiolo[4,5c]furan-4,6-dion with (i) $N$-(2-aminoethyl)ethane-1,2-diamine, (ii) heptane-1,7diamine, (iii) $N$-(3-aminopropyl)propane-1,3-diamine, (iv) octane-1,8-diamine

To a suspension of 2,2-dimethyl-dihydro-[1,3]dithiolo[4,5-c]furan-4,6-dion (500mg, 2.45 mmol) in methylene chloride $(12 \mathrm{ml})$ was added slowly a solution of $N$-(2-aminoethyl)ethane-1,2-diamine (155 mg, $1.5 \mathrm{mmol})$, heptane-1,7-diamine (195 mg, $1.5 \mathrm{mmol}$ ), $\mathrm{N}$-(3-aminopropyl)propane-1,3-diamine (197 mg, $1.5 \mathrm{mmol}$ ) or octane-1,8diamine $(216 \mathrm{mg}, 1.5 \mathrm{mmol})$ dissolved in methylene chloride $(1 \mathrm{ml})$ and stirred at $0^{\circ} \mathrm{C}$ under nitrogen. After stirring for 1 hour at room temperature the formed solid was collected and dried. This material was suitable for use without further purification.

\section{5,5'-[iminobis(ethane-2,1-diyliminocarbonyl)]bis(2,2-dimethyl-1,3-dithiolane-4- carboxylic acid) (3a):}

Compound 3a was prepared from $N$-(2-aminoethyl)ethane-1,2-diamine according to the general procedure above to provide $630 \mathrm{mg}$ (quant.) of 3a as pale yellow solid: m.p. 120$124^{\circ} \mathrm{C}$. MS: pos. ESI: $[\mathrm{M}+\mathrm{H}]^{+}, \mathrm{m} / \mathrm{z}$ 511.9; neg. ESI: $\left.[\mathrm{M}-\mathrm{H}]\right]^{-}, \mathrm{m} / \mathrm{z}$ 509.9; Exact mass calcd for $\mathrm{C}_{18} \mathrm{H}_{29} \mathrm{~N}_{3} \mathrm{O}_{6} \mathrm{~S}_{4}: 511.69 .{ }^{1} \mathbf{H}-\mathbf{N M R}(\mathrm{DMSO}) / 399.95 \mathrm{MHz}: \delta=1.70\left(\mathrm{~s}, 3 \mathrm{H}, \mathrm{CH}_{3}\right.$ ), 1.73 (s, 3H, CH $\mathbf{H}_{3}$ ), 2.74-2.99 (m, 4H, 4 H, $2 \mathrm{CH}_{2}-\mathrm{NH}$ ), 3.29-3.39 (m, 4H, $2 \mathrm{CH}_{2}-\mathrm{NH}-$ $\mathrm{C}=\mathrm{O}$ ), 4.39-4.44 (m, 2H, 2 CH-S), 4.78-4.83 (m, 2H, CH-S), 8.13 (t, ${ }^{3} \mathrm{~J}=5.6 \mathrm{~Hz}, 2 \mathrm{H}, 2$ $\mathrm{O}=\mathrm{C}-\mathrm{NH})$, amine proton was not detected

\section{5,5'-[heptane-1,7-diylbis(iminocarbonyl)]bis(2,2-dimethyl-1,3-dithiolane-4- carboxylic acid) (3b):}

Compound $\mathbf{3 b}$ was prepared from heptane-1,7-diamine according to the general procedure above to provide $660 \mathrm{mg}$ (quant.) of $3 \mathbf{b}$ as white solid: m.p. $50-53^{\circ} \mathrm{C}$. MS: pos. ESI: $[\mathrm{M}+\mathrm{H}]^{+}, \mathrm{m} / \mathrm{z}$ 539,3; neg. ESI: $[\mathrm{M}-\mathrm{H}]^{-}, \mathrm{m} / \mathrm{z}$ 537,2; Exact mass calcd for $\mathrm{C}_{21} \mathrm{H}_{34} \mathrm{~N}_{2} \mathrm{O}_{6} \mathrm{~S}_{4}: 538.76 .{ }^{1} \mathbf{H}-\mathbf{N M R}:\left(\mathrm{CDCl}_{3}\right) / 399,95 \mathrm{MHz}: \delta=1,23-1,71\left(\mathrm{~m}, 10 \mathrm{H}, 5 \mathrm{CH}_{2}\right)$;

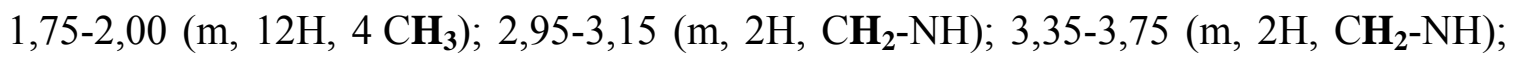
$\delta=4,60-4,83(\mathrm{~m}, 4 \mathrm{H}, 4 \mathrm{CH}-\mathrm{S}) ; \delta=6,98-7,21(\mathrm{~m}, 2 \mathrm{H}, 2 \mathrm{NH}) ; \delta=7,31-7,43(\mathrm{~m}, 2 \mathrm{H}$, $2 \mathrm{COOH})$ 
5,5'-[iminobis(propane-3,1-diyliminocarbonyl)]bis(2,2-dimethyl-1,3-dithiolane-4carboxylic acid) (3c):

Compound 3c was prepared from $N$-(3-aminopropyl)propane-1,3-diamine according to the general procedure above to provide $659 \mathrm{mg}$ (quant.) of 3c as pale yellow solid: m.p. $131-135^{\circ} \mathrm{C}$. MS: pos. ESI: $[\mathrm{M}+\mathrm{H}]^{+}, \mathrm{m} / \mathrm{z}$ 540,1; neg. ESI: [M-H] ${ }^{-}, \mathrm{m} / \mathrm{z}$ 538,2; Exact mass calcd for $\mathrm{C}_{20} \mathrm{H}_{33} \mathrm{~N}_{3} \mathrm{O}_{6} \mathrm{~S}_{4}:$ 539.75. ${ }^{1} \mathrm{H}$-NMR: (DMSO)/ 399,95MHz: $\delta=1,60-1,85$ (m, $16 \mathrm{H}, 4 \mathrm{CH}_{3}+2 \mathrm{CH}_{2}-\mathrm{CH}_{2}-\mathrm{CH}_{2}$ ); 2,82-3,28 (m, 8H, $\left.4 \mathrm{CH}_{2}-\mathrm{NH}\right) ; 4,21-4,79$ (m, 4H, 4 CH$\mathrm{S}) ; 7,75-8,05$ (m, 2H, $2 \mathrm{O}=\mathrm{C}-\mathrm{NH}) ; 8,45-9,50(\mathrm{~m}, 2 \mathrm{H}, 2 \mathrm{COOH})$, amine proton was not detected; ${ }^{13}$ C-NMR: $\left(\mathrm{CDCl}_{3}\right) / 100,57 \mathrm{MHz}: \delta=26,5\left(2 \mathrm{CH}_{2}-\mathrm{CH}_{2}-\mathrm{CH}_{2}\right) ; 31,7\left(2 \mathrm{C}-\mathrm{CH}_{3}\right)$; $36,7$ (2 C-CH $)$ ); 45,4 (2 $\left.\mathrm{CH}_{2}-\mathrm{NH}-\mathrm{CO}\right) ; 57,3(2 \mathrm{CH}-\mathrm{COOH}) ; 58,9\left(2 \mathrm{CH}_{2}-\mathrm{NH}-\mathrm{CH}_{2}\right) ; 61,0$ (4 CH-CO-NH); 63,0 ( $\left.\mathrm{CH}_{3}-\mathrm{C}-\mathrm{CH}_{3}\right) ; 172,4(2 \mathrm{CO}-\mathrm{NH}) ; 172,93(2 \mathrm{COOH})$

\section{5,5'-[octane-1,8-diylbis(iminocarbonyl)]bis(2,2-dimethyl-1,3-dithiolane-4-carboxylic} acid) (3d):

Compound 3d was prepared from octane-1,8-diamine according to the general procedure above to provide $679 \mathrm{mg}$ (quant.) of $\mathbf{3 d}$ as white solid: m.p. 115-118 ${ }^{\circ} \mathrm{C}$. MS: pos. ESI: $[\mathrm{M}+\mathrm{H}]^{+}, \mathrm{m} / \mathrm{z}$ 553.28; neg. ESI: [M-H] $]^{-} \mathrm{m} / \mathrm{z}$ 550.9. Anal. Calcd for $\mathrm{C}_{22} \mathrm{H}_{36} \mathrm{~N}_{2} \mathrm{O}_{6} \mathrm{~S}_{4}$ (Exact mass calcd: 552.79): C, 47.80; H, 6.56; N, 5.07; S, 23.20. Found: C, 47.79; H, 6.79; N, 5.37; S, 23.03. ${ }^{1} \mathbf{H}-\mathbf{N M R}$ (DMSO)/399.95 MHz: $\delta=1.15-1.20$ (m, 8H, $4 \mathrm{CH}_{2}$ alkyl), $1.30-$ 1.35 (m, 4H, $2 \mathrm{CH}_{2}$ alkyl), 1.67 (s, 3H, CH $\left.\mathbf{H}_{3}\right), 1.73$ (s, 3H, $\left.\mathbf{C H}_{3}\right), 2.93-2.99$ (m, 4H, 2 $\left.\mathrm{CH}_{2}-\mathrm{NH}-\mathrm{C}=\mathrm{O}\right), 4.43\left(\mathrm{~d},{ }^{3} \mathrm{~J}=5.6 \mathrm{~Hz}, 2 \mathrm{H}, 2\right.$ HOOC-CH-S$), 4.84$ (d, ${ }^{3} \mathrm{~J}=5.6 \mathrm{~Hz}, 2 \mathrm{H}, 2 \mathrm{NOC}-$ CH-S), $7.73\left(\mathrm{t},{ }^{3} \mathrm{~J}=5.6 \mathrm{~Hz}, 2 \mathrm{H}, 2 \mathrm{O}=\mathrm{C}-\mathrm{NH}\right), 12.68(\mathrm{~s}, 2 \mathrm{H}, 2 \mathrm{COOH})$

General procedure for the reaction of 4-nitrophenyl 5-[(diisobutylamino)carbonyl]2,2-dimethyl-1,3-dithiolane-4-carboxylate with (i) pentane-1,5-diamine, (ii) $\mathrm{N}$-(2aminoethyl)ethane-1,2-diamine, (iii) $N$-(3-aminopropyl)propane-1,3-diamine

To a solution of $2(134 \mathrm{mg}, 0.29 \mathrm{mmol})$ in toluene $(5 \mathrm{ml})$ was added slowly a solution of pentane-1,5-diamine (30 mg, $0.29 \mathrm{mmol}), \mathrm{N}$-(2-aminoethyl)ethane-1,2-diamine (30 $\mathrm{mg}$, $0.29 \mathrm{mmol}$ ) or $\mathrm{N}$-(3-aminopropyl)propane-1,3-diamine (38 $\mathrm{mg}, 0.29 \mathrm{mmol}$ ) dissolved in toluene $(1 \mathrm{ml})$ and stirred at room temperature. A byproduct precipitates, that after 
stirring for 1 hour at room temperature was collected and discarded. The mother liquor was concentrated in vacuo to yield a pale yellow viscous oil. This material was suitable for use without further purification.

\section{$N^{5}, N^{5}$-pentane-1,5-diylbis( $N, N$-diisobutyl-2,2-dimethyl-1,3-dithiolane-4,5- dicarboxamide) (3e):}

Compound 3e was prepared from pentane-1,5-diamine according to the general procedure above to provide $106 \mathrm{mg}$ (quant.) of $\mathbf{3 e}$ as pale yellow oil. The oil was dissolved in ether and after slow evaporation a pale yellow solid precipitated: m.p. 140$145^{\circ} \mathrm{C}$. MS: pos. ESI: $[\mathrm{M}+\mathrm{H}]^{+}, \mathrm{m} / \mathrm{z}$ 733.3, $[\mathrm{M}+\mathrm{Na}]^{+}, \mathrm{m} / \mathrm{z} 755.1$; neg. ESI: $\left.[\mathrm{M}-\mathrm{H}]\right]^{-}, \mathrm{m} / \mathrm{z}$ 731.2; Anal. Calcd for $\mathrm{C}_{35} \mathrm{H}_{64} \mathrm{~N}_{4} \mathrm{O}_{4} \mathrm{~S}_{4}$ (Exact mass calcd: 733.17): C, 57.34; $\mathrm{H}, 8.80$; N, 7.64; S, 17.49. Found: C, 56.95; H, 8.90; N, 7.76; S, 17.31. ${ }^{1} \mathbf{H}-\mathrm{NMR}\left(\mathrm{CDCl}_{3}\right) / 399.95$ MHz: $\delta=0.83-0.87\left(\mathrm{~m}, 12 \mathrm{H}, 4 \mathrm{CH}_{3}-\mathrm{CH}\right), 0.92-0.96\left(\mathrm{~m}, 12 \mathrm{H}, 4 \mathrm{CH}_{3}-\mathrm{CH}\right), 1.31-1.35(\mathrm{~m}$, $2 \mathrm{H}, \mathrm{CH}_{2}-\mathrm{CH}_{2}-\mathrm{CH}_{2}$ ), 1.45-1.53 (m, 4H, $\mathrm{CH}_{2}-\mathrm{CH}_{2}-\mathrm{CH}_{2}$ ), 1.83 (s, 3H, $\mathrm{CH}_{3}$ ), 1.86 (s, 3H, $\mathrm{CH}_{3}$ ), 1.91-2.01 (m, 4H, $4 \mathrm{CH}_{3}-\mathrm{CH}-\mathrm{CH}_{3}$ ), 3.03-3.14 (m, 8H, $4 \mathrm{CH}_{3}-\mathrm{CH}-\mathrm{CH}_{2}$ ), 3.21-3.32 (m, 4H, $\left.2 \mathrm{CH}_{2}-\mathrm{NH}-\mathrm{C}=\mathrm{O}\right), 4.62\left(\mathrm{~d},{ }^{3} \mathrm{~J}=5.6 \mathrm{~Hz}, 2 \mathrm{H}, 2 \mathrm{CH}-\mathrm{S}\right), 4.89\left(\mathrm{~d},{ }^{3} \mathrm{~J}=5.6 \mathrm{~Hz}, 2 \mathrm{H}\right.$, CH-S), 6.70-6.74 (m, 2N, 2 NH-C=O)

\section{$N^{5}, N^{5}$-(iminodiethane-2,1-diyl)bis( $N, N$-diisobutyl-2,2-dimethyl-1,3-dithiolane-4,5- dicarboxamide) (3f):}

Compound 3f was prepared from $N$-(2-aminoethyl)ethane-1,2-diamine according to the general procedure above to provide $107 \mathrm{mg}$ (quant.) of $\mathbf{3 f}$ as pale yellow oil. The oil was dissolved in ether and after slow evaporation a pale yellow solid precipitated: m.p. 120$125^{\circ} \mathrm{C}$. MS: pos. ESI: $[\mathrm{M}+\mathrm{H}]^{+}, \mathrm{m} / \mathrm{z}$ 734.1, $[\mathrm{M}+\mathrm{Na}]^{+}, \mathrm{m} / \mathrm{z}$ 756.2; neg. ESI: $\left.[\mathrm{M}-\mathrm{H}]\right]^{-}, \mathrm{m} / \mathrm{z}$ 732.1; Exact mass calcd for $\mathrm{C}_{34} \mathrm{H}_{63} \mathrm{~N}_{5} \mathrm{O}_{4} \mathrm{~S}_{4}$ : 734.15.

${ }^{1} \mathbf{H}-\mathrm{NMR}\left(\mathrm{CDCl}_{3}\right) / 399.95 \mathrm{MHz}: \delta=0.82-0.86\left(\mathrm{~m}, 12 \mathrm{H}, 4 \mathrm{CH}_{3}-\mathrm{CH}\right), 0.91-0.95(\mathrm{~m}, 12 \mathrm{H}$, $4 \mathrm{CH}_{3}-\mathrm{CH}$ ), 1.82 (s, 3H, CH $\left.\mathbf{H}_{3}\right), 1.86\left(\mathrm{~s}, 3 \mathrm{H}, \mathrm{CH}_{3}\right), 1.90-2.01$ (m, 4H, $4 \mathrm{CH}_{3}-\mathrm{CH}-\mathrm{CH}_{3}$ ), 2.73-2.82 (m, 6H, CH $\left.\mathbf{H}_{2}-\mathrm{NH}-\mathrm{CH}_{2}, 2 \mathrm{CH}_{2}-\mathrm{NH}-\mathrm{C}=\mathrm{O}\right), 3.07-3.41$ (m, $10 \mathrm{H}, 4 \mathrm{CH}_{3}-\mathrm{CH}-\mathrm{CH}_{2}$, $\left.2 \mathrm{CH}_{2}-\mathrm{NH}-\mathrm{C}=\mathrm{O}\right), 4.60\left(\mathrm{~d},{ }^{3} \mathrm{~J}=5.7 \mathrm{~Hz}, 2 \mathrm{H}, 2 \mathrm{CH}-\mathrm{S}\right), 4.88\left(\mathrm{~d},{ }^{3} \mathrm{~J}=5.7 \mathrm{~Hz}, 2 \mathrm{H}, \mathrm{CH}-\mathrm{S}\right), 6.95-$ $7.01(\mathrm{~m}, 2 \mathrm{~N}, 2 \mathrm{NH}-\mathrm{C}=\mathrm{O})$, amine proton was not detected. 
$N^{5}, N^{5}$-(iminodipropane-3,1-diyl)bis( $N, N$-diisobutyl-2,2-dimethyl-1,3-dithiolane-4,5dicarboxamide) (3g):

Compound 3g was prepared from $N$-(3-aminopropyl)propane-1,3-diamine according to the general procedure above to provide $112 \mathrm{mg}$ (quant.) of $\mathbf{3 g}$ as pale yellow oil. MS: pos. ESI: $[\mathrm{M}+\mathrm{H}]^{+}, \mathrm{m} / \mathrm{z}$ 762.62; neg. ESI: $[\mathrm{M}-\mathrm{H}]^{-}, \mathrm{m} / \mathrm{z}$ 760.49; Exact mass calcd for $\mathrm{C}_{34} \mathrm{H}_{63} \mathrm{~N}_{5} \mathrm{O}_{4} \mathrm{~S}_{4}:$ 734.15. ${ }^{1} \mathbf{H}-\mathbf{N M R}\left(\mathrm{CDCl}_{3}\right) / 399.95 \mathrm{MHz}: \delta=0.83-0.86\left(\mathrm{~m}, 12 \mathrm{H}, 4 \mathrm{CH}_{3^{-}}\right.$ $\mathrm{CH}), 0.90-0.93$ (m, 12H, $\left.4 \mathrm{CH}_{3}-\mathrm{CH}\right), 1.60-1.68$ (m, 4H, $\left.2 \mathrm{CH}_{2}-\mathrm{CH}_{2}-\mathrm{CH}_{2}\right), 1.81$ (s, 3H, $\left.\mathrm{CH}_{3}\right), 1.85$ (s, 3H, $\left.\mathrm{CH}_{3}\right), 1.91-2.00\left(\mathrm{~m}, 4 \mathrm{H}, 4 \mathrm{CH}_{3}-\mathrm{CH}-\mathrm{CH}_{3}\right), 2.61-2.66\left(\mathrm{~m}, 4 \mathrm{H}, \mathrm{CH}_{2}-\right.$ $\left.\mathrm{NH}-\mathrm{CH}_{2}\right), 3.00-3.10\left(\mathrm{~m}, 6 \mathrm{H}, 4 \mathrm{CH}_{3}-\mathrm{CH}-\mathrm{CH}_{2}, 2 \mathrm{CH}_{2}-\mathrm{NH}-\mathrm{C}=\mathrm{O}\right), 3.21-3.31$ (m, 6H, $\left.4 \mathrm{CH}_{3}-\mathrm{CH}-\mathrm{CH}_{2}, 2 \mathrm{CH}_{2}-\mathrm{NH}-\mathrm{C}=\mathrm{O}\right), 4.64\left(\mathrm{~d},{ }^{3} \mathrm{~J}=5.6 \mathrm{~Hz}, 2 \mathrm{H}, 2 \mathrm{CH}-\mathrm{S}\right), 4.88\left(\mathrm{~d},{ }^{3} \mathrm{~J}=5.6 \mathrm{~Hz}\right.$, 2H, CH-S), 7.15-7.21 (m, 2N, 2 NH-C=O), amine proton was not detected. ${ }^{13} \mathbf{C}-\mathbf{N M R}$ $\left(\mathrm{CDCl}_{3}\right) / 100.57 \mathrm{MHz}: \delta=20.3\left(4 \mathrm{CH}_{3}-\mathrm{CH}\right), 20.4\left(2 \mathrm{CH}_{3}-\mathrm{CH}\right), 20.5\left(2 \mathrm{CH}_{3}-\mathrm{CH}\right), 26.7$ (2 $\left.\mathrm{CH}_{3}-\mathrm{CH}\right), 28.2\left(2 \mathrm{CH}_{3}-\mathrm{CH}\right), 29.2\left(\mathrm{CH}_{2}-\mathrm{CH}_{2}-\mathrm{CH}_{2}\right), 29.3\left(\mathrm{CH}_{2}-\mathrm{CH}_{2}-\mathrm{CH}_{2}\right), 32.7\left(\mathrm{CH}_{3}\right)$, $32.8\left(\mathrm{CH}_{3}\right), 36.2\left(2 \mathbf{C H}_{3}\right), 38.8\left(\mathrm{CH}_{2}-\mathrm{CH}\right), 38.9\left(\mathrm{CH}_{2}-\mathrm{CH}\right), 48.0\left(\mathrm{NH}-\mathrm{CH}_{2}\right), 48.1(\mathrm{NH}-$ $\left.\mathrm{CH}_{2}\right), 53.9$ (2 $\left.\mathbf{C H}_{2}-\mathrm{NH}-\mathrm{C}=\mathrm{O}\right), 56.2\left(2 \mathrm{CH}_{2}-\mathrm{CH}\right), 56.5$ (CH-S), 56.6 (CH-S), 60.6 (CH-S), 60.7 (CH-S), 62.1 (S-C-S), 62.2 (S-C-S), 168.5 (N-C=O), 168.7 (N-C=O)

\section{Synthesis of 4,4'-[iminobis(ethane-2,1-diylimino)]bis(2,3-dimercapto-4-oxobutanoic acid) (4a):}

To a solution of 3a (341 mg, $0.66 \mathrm{mmol})$ in acetonitrile/water (3:1) ( $5 \mathrm{ml})$ was added slowly a solution of $\mathrm{HgCl}_{2}(1790 \mathrm{mg}, 6.6 \mathrm{mmol})$ dissolved in acetonitrile/water (3:1) (20 $\mathrm{ml}$ ) and stirred at room temperature. The resulting mercury complex precipitates. After stirring for 2 hours at room temperature the solvent mixture was evaporated to dryness. The white solid was washed with water, collected by filtration and dried. The dry solid was suspended in acetonitrile/water (3:1) (50ml) and hydrogen sulphide was bubbled into the stirring suspension. Step by step the mercury complex dissolved and $\mathrm{HgS}$ precipitated. After 1 hour the gas stream was stopped, the black precipitate was collected by filtration and disposed.

The mother liquor was concentrated in vacuo to yield $256 \mathrm{mg}(90 \%)$ of $\mathbf{4 a}$ as colourless viscous oil. This crude product was used for chelating reaction without purification. 
MS: pos. ESI: $[\mathrm{M}+\mathrm{H}]^{+}, \mathrm{m} / \mathrm{z}$ 431.8, [M+Na] $]^{+}, \mathrm{m} / \mathrm{z}$ 453.9; neg. ESI: [M-H] $]^{-}, \mathrm{m} / \mathrm{z}$ 429.8;

Exact mass calcd for $\mathrm{C}_{12} \mathrm{H}_{21} \mathrm{~N}_{3} \mathrm{O}_{6} \mathrm{~S}_{4}: 431.57$.

\section{4,4'-(heptane-1,7-diyldiimino)bis(2,3-dimercapto-4-oxobutanoic acid) (4b):}

Compound $\mathbf{4 b}$ was prepared from $\mathbf{3 b}$ according to the general procedure above to provide $257 \mathrm{mg}(85 \%)$ of $\mathbf{4 b}$ as pale yellow oil. MS: pos. ESI: $[\mathrm{M}+\mathrm{H}]^{+}, \mathrm{m} / \mathrm{z} 459.0,[\mathrm{M}+\mathrm{Na}]^{+}, \mathrm{m} / \mathbf{z}$ 481.0; neg. ESI: [M-H]', m/z 457.1; Exact mass calcd for $\mathrm{C}_{15} \mathrm{H}_{26} \mathrm{~N}_{2} \mathrm{O}_{6} \mathrm{~S}_{4}: 458.64$.

\section{4,4'-[iminobis(propane-3,1-diylimino)]bis(2,3-dimercapto-4-oxobutanoic acid) (4c):}

Compound $\mathbf{4} \mathbf{c}$ was prepared from $3 \mathbf{c}$ according to the general procedure above to provide $264 \mathrm{mg}(87 \%)$ of $4 \mathrm{c}$ as pale yellow oil. MS: pos. ESI: [M+H] $]^{+}, \mathrm{m} / \mathrm{z} 460.1,[\mathrm{M}+\mathrm{Na}]^{+}, \mathrm{m} / \mathrm{z}$ 482.2; neg. ESI: [M-H]', m/z 458.2; Exact mass calcd for $\mathrm{C}_{14} \mathrm{H}_{25} \mathrm{~N}_{3} \mathrm{O}_{6} \mathrm{~S}_{4}: 459.63$.

\section{4,4'-(octane-1,8-diyldiimino)bis(2,3-dimercapto-4-oxobutanoic acid) (4d):}

Compound $4 \mathbf{d}$ was prepared from $3 \mathbf{d}$ according to the general procedure above to provide $284 \mathrm{mg}(91 \%)$ of $\mathbf{4 d}$ as pale yellow oil. MS: pos. ESI: [M+H] $]^{+}, \mathrm{m} / \mathrm{z}$ 473.1; neg. ESI: [M$\mathrm{H}]^{-}, \mathrm{m} / \mathrm{z}$ 470.9; Exact mass calcd for $\mathrm{C}_{16} \mathrm{H}_{28} \mathrm{~N}_{2} \mathrm{O}_{6} \mathrm{~S}_{4}: 472.66$.

\section{$N^{2}, N^{2}$-pentane-1,5-diylbis( $\left(N^{4}, N^{4}\right.$-diisobutyl-2,3-dimercaptosuccinamide) (4e):}

Compound $4 \mathbf{e}$ was prepared from $3 \mathbf{e}$ according to the general procedure above to provide $366 \mathrm{mg}(85 \%)$ of $\mathbf{4 d}$ as pale yellow oil. Instead off acetonirile/water (3:1), methanol was used for suspending the mercury complex. MS: pos. ESI: $[\mathrm{M}+\mathrm{H}]^{+}, \mathrm{m} / \mathrm{z} 653.5,[\mathrm{M}+\mathrm{Na}]^{+}$, m/z 675.3 ; neg. ESI: [M-H]', m/z 651.1; Exact mass calcd for $\mathrm{C}_{29} \mathrm{H}_{56} \mathrm{~N}_{4} \mathrm{O}_{4} \mathrm{~S}_{4}$ : 653.04 . ${ }^{1} \mathbf{H}-\mathrm{NMR}\left(\mathrm{CDCl}_{3}\right) / 399.95 \mathrm{MHz}: \delta=0.87-0.91\left(\mathrm{~m}, 12 \mathrm{H}, 4 \mathrm{CH}_{3}-\mathrm{CH}\right), 0.97-0.99(\mathrm{~m}, 12 \mathrm{H}$, $\left.4 \mathrm{CH}_{3}-\mathrm{CH}\right), 1.45-1.54\left(\mathrm{~m}, 2 \mathrm{H}, \mathrm{CH}_{2}-\mathrm{CH}_{2}-\mathrm{CH}_{2}\right), 1.55-1.68$ (m, $4 \mathrm{H}, \mathrm{CH}_{2}-\mathrm{CH}_{2}-\mathrm{CH}_{2}$ ), 2.01-2.10 (m, 4H, $\left.4 \mathrm{CH}_{3}-\mathrm{CH}-\mathrm{CH}_{3}\right), 3.12-3.56\left(\mathrm{~m}, 12 \mathrm{H}, 4 \mathrm{CH}_{3}-\mathrm{CH}-\mathrm{CH}_{2}, 2 \mathrm{CH}_{2}-\mathrm{NH}-\right.$ $\mathrm{C}=\mathrm{O}), 4.34-4.46(\mathrm{~m}, 4 \mathrm{H}, 4 \mathrm{CH}-\mathrm{S}), 8.68-8.75(\mathrm{~m}, 2 \mathrm{H}, 2 \mathrm{NH}-\mathrm{C}=\mathrm{O})$, thiol protons were not detected. 
$N^{2}, N^{2}$-(iminodiethane-2,1-diyl)bis $\left(N^{4}, N^{4}\right.$-diisobutyl-2,3-dimercaptosuccinamide) (4f):

Compound $\mathbf{4 f}$ was prepared from $3 \mathbf{f}$ according to the general procedure above to provide $380 \mathrm{mg}(88 \%)$ of $\mathbf{4 f}$ as pale yellow oil. Instead off acetonirile/water (3:1), methanol was used for suspending the mercury complex.

MS: pos. ESI: $[\mathrm{M}+\mathrm{H}]^{+}, \mathrm{m} / \mathrm{z}$ 654.3, [M+Na] $]^{+}, \mathrm{m} / \mathrm{z} 676.4$; neg. ESI: [M-H] $]^{-}$m/z 652.2;

Exact mass calcd for $\mathrm{C}_{28} \mathrm{H}_{55} \mathrm{~N}_{5} \mathrm{O}_{4} \mathrm{~S}_{4}: 654.03$. ${ }^{1} \mathbf{H}-\mathrm{NMR}\left(\mathrm{CDCl}_{3}\right) / 399.95 \mathrm{MHz}: \delta=0.86-$

0.90 (m, 12H, $\left.4 \mathrm{CH}_{3}-\mathrm{CH}\right), 0.95-0.99\left(\mathrm{~m}, 12 \mathrm{H}, 4 \mathrm{CH}_{3}-\mathrm{CH}\right), 1.90-2.01$ (m, 4H, $4 \mathrm{CH}_{3}-\mathrm{CH}-$

$\mathrm{CH}_{3}$ ), 2.35-2.52 (m, 4H, CH $\mathbf{H}_{2}-\mathrm{NH}-\mathrm{CH}_{2}$ ), 3.03-3.47 (m, 12H, $4 \mathrm{CH}_{3}-\mathrm{CH}-\mathrm{CH}_{2}, 2 \mathrm{CH}_{2}-\mathrm{NH}-$ $\mathrm{C}=\mathrm{O}), 4.53\left(\mathrm{~d},{ }^{3} \mathrm{~J}=5.6 \mathrm{~Hz}, 2 \mathrm{H}, 2 \mathrm{CH}-\mathrm{S}\right), 4.88\left(\mathrm{~d},{ }^{3} \mathrm{~J}=5.6 \mathrm{~Hz}, 2 \mathrm{H}, \mathrm{CH}-\mathrm{S}\right), 6.95-7.01$ (m, $2 \mathrm{~N}, 2 \mathrm{NH}-\mathrm{C}=\mathrm{O}$ ), amine and thiol protons were not detected.

$N^{2}, N^{2}$-(iminodipropane-3,1-diyl)bis $\left(N^{4}, N^{4}\right.$-diisobutyl-2,3-dimercaptosuccinamide) (4g):

Compound $\mathbf{4 g}$ was prepared from $\mathbf{3 g}$ according to the general procedure above to provide $405 \mathrm{mg}(90 \%)$ of $\mathbf{4 g}$ as pale yellow oil. Instead off acetonirile/water (3:1), methanol was used for suspending the mercury complex. MS: pos. ESI: $[\mathrm{M}+\mathrm{H}]^{+}, \mathrm{m} / \mathrm{z} 682.6$; neg. ESI: $[\mathrm{M}-\mathrm{H}]^{-}, \mathrm{m} / \mathrm{z}$ 680.3; Exact mass calcd for $\mathrm{C}_{30} \mathrm{H}_{59} \mathrm{~N}_{5} \mathrm{O}_{4} \mathrm{~S}_{4}:$ 682.08. ${ }^{1} \mathbf{H}-\mathbf{N M R}\left(\mathrm{CDCl}_{3}\right) /$ 399.95 MHz: $\delta=0.84-0.98\left(\mathrm{~m}, 24 \mathrm{H}, 4 \mathrm{CH}_{3}-\mathrm{CH}\right), 1.95-2.11\left(\mathrm{~m}, 8 \mathrm{H}, 2 \mathrm{CH}_{2}-\mathrm{CH}_{2}-\mathrm{CH}_{2}, 4\right.$ $\mathrm{CH}_{3}-\mathrm{CH}-\mathrm{CH}_{3}$ ), 2.42 ( d, $\left.{ }^{3} \mathrm{~J}=10.2 \mathrm{~Hz}, 2 \mathrm{H}, 2 \mathrm{SH}\right), 2.44$ ( d, ${ }^{3} \mathrm{~J}=10.2 \mathrm{~Hz}, 2 \mathrm{H}, 2 \mathrm{SH}$ ), 3.01 $3.62\left(\mathrm{~m}, 16 \mathrm{H}, \mathrm{CH}_{2}-\mathrm{NH}-\mathrm{CH}_{2}, 4 \mathrm{CH}_{3}-\mathrm{CH}-\mathrm{CH}_{2}, 2 \mathrm{CH}_{2}-\mathrm{NH}-\mathrm{C}=\mathrm{O}\right), 3.86\left(\mathrm{dd},{ }^{3} \mathrm{~J}=10.2,9.8\right.$ $\mathrm{Hz}, 2 \mathrm{H}, 2 \mathrm{CH}-\mathrm{S}$ ), 3.94 (dd, $\left.{ }^{3} \mathrm{~J}=9.8,10.2 \mathrm{~Hz}, 2 \mathrm{H}, \mathrm{CH}-\mathrm{S}\right), 7.82\left(\mathrm{t},{ }^{3} \mathrm{~J}=6.0 \mathrm{~Hz}, 2 \mathrm{H}, 2 \mathrm{NH}-\right.$ $\mathrm{C}=\mathrm{O}$ ), amine proton was not detected. 


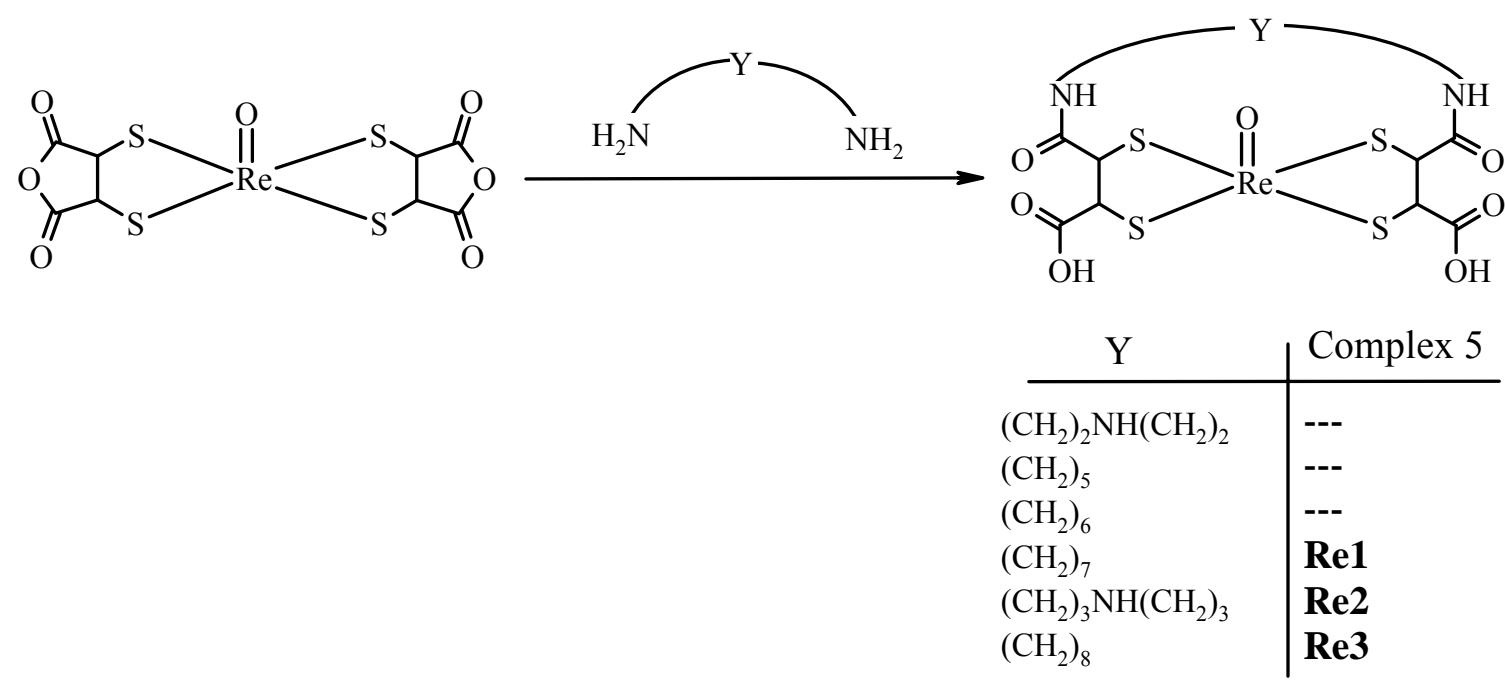

Scheme 2. Reaction of a alkylene diamines with $\mathrm{ReO}(\mathrm{DMSA} \text {-anhydride })_{2}$ to form complexes $\mathbf{R e 1}-\mathbf{R e} 3$

General procedure for the reaction of tetrabutylammonium tetrachlorooxorhenate(V) with $4 \mathrm{~b}, 4 \mathrm{c}$, $4 \mathrm{e}$ or $\mathbf{4 g}$.

To a solution of tetrabutylammonium tetrachlorooxorhenate(V) $(10 \mathrm{mg}, 0.02 \mathrm{mmol})$ in dry methanol $(10 \mathrm{ml})$ was added slowly a solution of $\mathbf{4 b}(8 \mathrm{mg}, 0.02 \mathrm{mmol}), \mathbf{4 c}(8 \mathrm{mg}$, 0,02 mmol), $4 \mathbf{e}(11 \mathrm{mg}, 1.5 \mathrm{mmol})$ or $\mathbf{4 g}(12 \mathrm{mg}, 0.02 \mathrm{mmol})$ dissolved dry methanol (10 $\mathrm{ml}$ ) and stirred at room temperature under nitrogen. The prior green solution changed the colour to orange. After stirring for 1 hour the solvent was removed in vacuo to give brown oils. This crude product was analyzed by ESI-MS.

\section{Tetrabutylammonium 4,4'-(heptane-1,7-diyldiimino)bis(2,3-dithiolato-4-} oxobutanoic acid)-(S,S,S,S) oxorhenate(V) (Re1):

Compound Re1 was prepared from $\mathbf{4 b}$ according to the general procedure above. MS: pos. ESI: $[\mathrm{M}+2 \mathrm{H}]^{+}, \mathrm{m} / \mathrm{z}$ 658.8; neg. ESI: $[\mathrm{M}]^{-}, \mathrm{m} / \mathrm{z}$ 657.1; Exact mass calcd for $\mathrm{C}_{15} \mathrm{H}_{22} \mathrm{~N}_{2} \mathrm{O}_{7} \mathrm{ReS}_{4}$ : 656.81 . 


\section{4,4'-[Iminiumbis(propane-3,1-diylimino)]bis(2,3-dithiolato-4-oxobutanoic acid)- (S,S,S,S) oxorhenate(V) (Re2):}

Compound Re2 was prepared from 4c according to the general procedure above. MS: pos. ESI: $[\mathrm{M}+\mathrm{H}]^{+}, \mathrm{m} / \mathrm{z}$ 660.1; neg. ESI: [M-H] $]^{-} \mathrm{m} / \mathrm{z}$ 658.0; Exact mass calcd for $\mathrm{C}_{14} \mathrm{H}_{21} \mathrm{~N}_{3} \mathrm{O}_{7} \mathrm{ReS}_{4}: 658.80$.

Tetrabutylammonium $N^{2}, N^{2}$-pentane-1,5-diylbis $\left(N^{4}, N^{4}\right.$-diisobutyl-2,3dithiolatosuccinamide)-(S,S,S,S) oxorhenate(V) (Re4):

Compound Re4 was prepared from $4 \mathbf{e}$ according to the general procedure above. MS: pos. ESI: $[\mathrm{M}+2 \mathrm{H}]^{+}, \mathrm{m} / \mathrm{z}$ 853.2; neg. ESI: [M] ${ }^{-}, \mathrm{m} / \mathrm{z}$ 851.2; Exact mass calcd. for $\mathrm{C}_{29} \mathrm{H}_{52} \mathrm{~N}_{4} \mathrm{O}_{5} \mathrm{ReS}_{4}: 851.21$.

General procedure for the reaction of $\mathrm{NBu}_{4}\left[\mathrm{ReO}(\mathrm{dmsaa})_{2}\right]$ [19] with (i) heptane-1,7diamine, (ii) $N$-(3-aminopropyl)propane-1,3-diamine, (iii) octane-1,8-diamine

$\mathrm{NBu}_{4}\left[\mathrm{ReO}(\mathrm{dmsaa})_{2}\right](5 \mathrm{mg}, 6.5 \mu \mathrm{mol})$ was dissolved in acetonitrile $(5 \mathrm{ml})$ and heptane1,7-diamine (1 mg, $6.5 \mu \mathrm{mol}$ ), $N$-(3-aminopropyl)propane-1,3-diamine (1 mg, $6.5 \mu \mathrm{mol})$ or octane-1,8-diamine $(1 \mathrm{mg}, 6.5 \mu \mathrm{mol})$ dissolved in acetonitrile $(1 \mathrm{ml})$ was added whilst stirring to give immediately an orange or brown solution.

This was stirred $1 \mathrm{~h}$ at room temperature and evaporated under vacuo and the residue $5 \mathbf{b}$ d analysed by ESI-MS.

\section{Tetrabutylammonium 4,4'-(heptane-1,7-diyldiimino)bis(2,3-dithiolato-4- oxobutanoic acid)-(S,S,S,S) oxo-rhenate(V) (Re1):}

Compound $\mathbf{R e} 1$ was prepared from $\mathrm{NBu}_{4}\left[\mathrm{ReO}(\text { dmsaa })_{2}\right]$ according to the general procedure above. MS: pos. ESI: [M+2H] $]^{+}, \mathrm{m} / \mathrm{z}$ 658.8; neg. ESI: [M]', m/z 657.1; Exact mass calcd for $\mathrm{C}_{15} \mathrm{H}_{22} \mathrm{~N}_{2} \mathrm{O}_{7} \mathrm{ReS}_{4}$ : 656.81. 


\section{4,4'-[Iminiumbis(propane-3,1-diylimino)]bis(2,3-dithiolato-4-oxobutanoic acid)- (S,S,S,S) oxo-rhenate(V) (Re2):}

Compound $\mathbf{R e} 2$ was prepared from $\mathrm{NBu}_{4}\left[\mathrm{ReO}(\text { dmsaa })_{2}\right]$ according to the general

procedure above. MS: pos. ESI: $[\mathrm{M}+\mathrm{H}]^{+}, \mathrm{m} / \mathrm{z}$ 660.1; neg. ESI: [M-H] $]^{-}, \mathrm{m} / \mathrm{z}$ 658.0; Exact mass calcd for $\mathrm{C}_{14} \mathrm{H}_{21} \mathrm{~N}_{3} \mathrm{O}_{7} \mathrm{ReS}_{4}$ : 658.80.

\section{Tetrabutylammonium 4,4'-(octane-1,8-diyldiimino)bis(2,3-dithiolato-4-oxobutanoic} acid)-(S,S,S,S) oxo-rhenate(V) (Re3):

Compound $\mathbf{R e} 3$ was prepared from $\mathrm{NBu}_{4}\left[\mathrm{ReO}(\text { dmsaa })_{2}\right]$ according to the general procedure above. MS: pos. ESI: $[\mathrm{M}+2 \mathrm{H}]^{+}, \mathrm{m} / \mathrm{z}$ 673.0; neg. ESI: [M]', m/z 671.1; Exact mass calcd for $\mathrm{C}_{16} \mathrm{H}_{24} \mathrm{~N}_{2} \mathrm{O}_{7} \mathrm{ReS}_{4}: 670.84$. 


\section{Uv/vis spectroscopical characterization of Re5a and Re5b}

Solutions $\left(10^{-5} \mathrm{M}\right)$ of the two isomers were prepared in acetonitrile. Relatively identical electronic absorption spectra were obtained for complexes Re5a and Re5b with a distinct (specifiable) maximum at $330 \mathrm{~nm}(\lg \varepsilon=3.3)$ and $340 \mathrm{~nm}(\lg \varepsilon=3.3)$ due to a ligand-tometal charge transfer (LMCT) transition (Figure 1). The intense absorptions at ca. 200$240 \mathrm{~nm}$ are related to intraligand (IL) transitions $\left(\pi-\pi^{*}\right)$ since similar absorption bands are observed for the uncoordinated ligands. UV-Vis spectra were recorded in acetonitrile using a Specord S10 spectrometer (Zeiss).

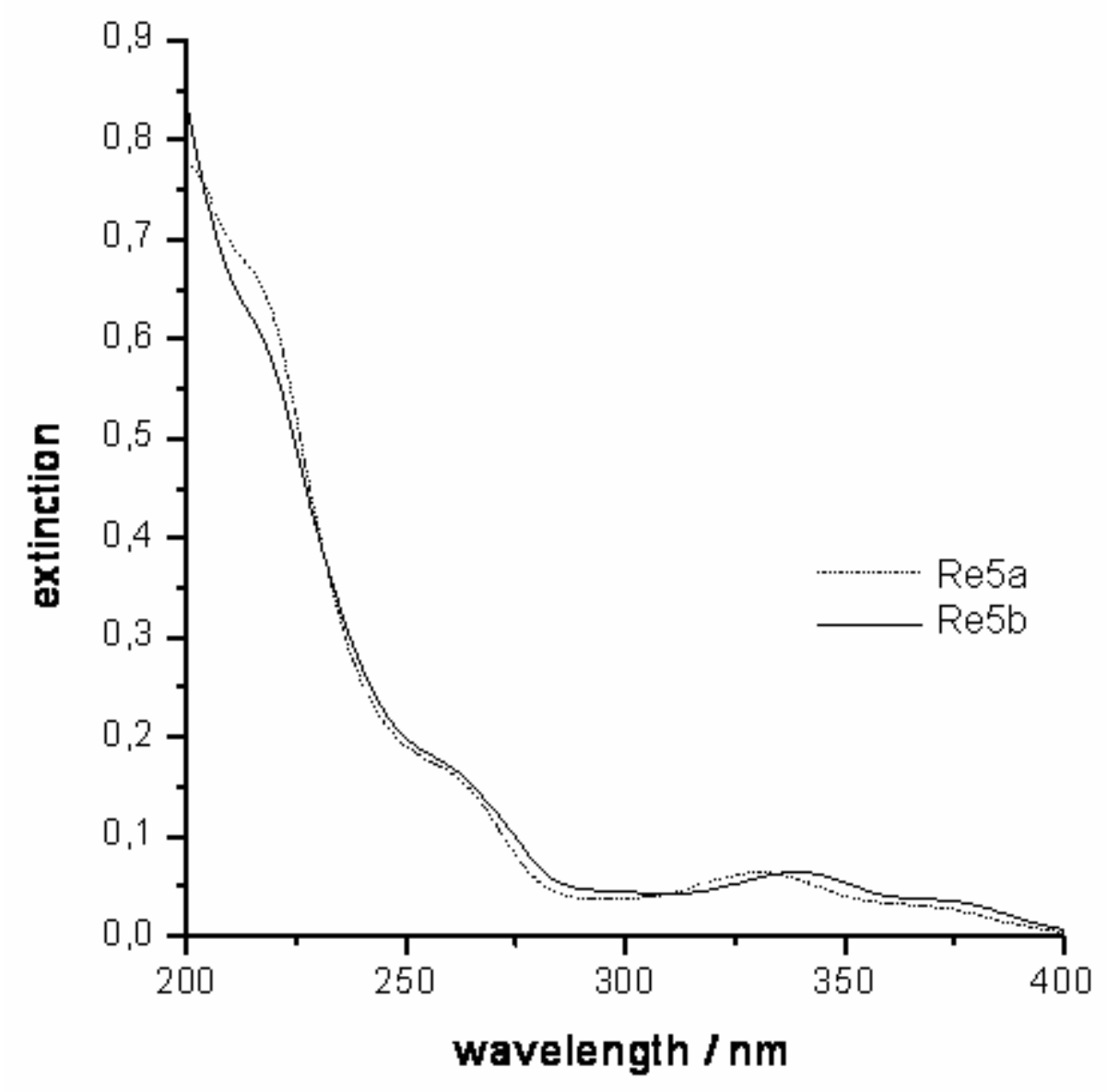

Figure 1. Absorption spectra of the exo-cis- (Re5a) and exo-trans-isomer (Re5b). 